\title{
Accurate mapping of chromatic dispersion distribution along optical fibres with spatial resolution below $100 \mathrm{~m}$
}

\author{
Kwang-Yong Song, Miguel González Herráez and Luc Thévenaz \\ Ecole Polytechnique Fédérale de Lausanne, Nanophotonics and Metrology Laboratory, Section 11, \\ CH-1015, Lausanne, Switzerland
}

\begin{abstract}
We demonstrate experimentally a threefold increase in the best resolution ever reported in distributed measurements of chromatic dispersion along single-mode optical fibres. Our method is based on probing the four-wave mixing efficiency along the fiber using Brillouin Optical Time Domain Analysis. We achieve record resolutions of $60 \mathrm{~m}$ in a $1.5 \mathrm{~km}$ standard fibre and $150 \mathrm{~m}$ in a $6.7 \mathrm{~km}$ dispersion shifted fibre, respectively.
\end{abstract}

Keywords: Chromatic dispersion, Brillouin shift, Brillouin Optical Time Domain Analysis

\section{INTRODUCTION}

Precise information of chromatic dispersion distribution along optical fibers has been shown to be beneficial in experiments related to massive wavelength division multiplexing (WDM) and dispersion-managed soliton transmission [1]. Additionally, recent studies concerning fiber-optic parametric amplifiers and wavelength converters show that dispersion fluctuations might be strongly detrimental in these devices if they are uncontrolled [2] or highly beneficial if they are adequately designed and tailored [3]. As a result, several methods for mapping the variation of the chromatic dispersion along the fiber have already been reported in the literature [4-6]. In particular, the method reported in [6] using four-wave mixing and Brillouin Optical Time Domain Analysis (BOTDA) has shown explicit advantages over the others because it can be used in the wavelength region of small anomalous dispersion, where its applications are extremely interesting.

As for the spatial resolution of the measurement, the best resolutions reported up to now have been $\sim 300 \mathrm{~m}$ in the case of standard fibres and $\sim 500 \mathrm{~m}$ in dispersion-shifted fibres (DSFs). These figures might be adequate for the characterization of soliton-based fiber links, but they are still too large for nonlinear devices (parametric amplifiers and wavelength converters) requiring high-resolution dispersion mapping in short lengths of fibre of only $1-2 \mathrm{~km}$.

In this paper, we present a record performance in the dispersion mapping of optical fibers using a highly improved version of the method reported in [6]. We achieve spatial resolutions of $60 \mathrm{~m}$ in a standard fibre and $150 \mathrm{~m}$ in a dispersion-shifted fibre, which are, to our knowledge, the highest resolutions ever reported. We also discuss limiting factors in the spatial resolution improvement using this technique.

\section{PRINCIPLE}

When two moderately powerful pump waves $\left(f_{I}<f_{K}\right)$ copropagate along an optical fibre, the process of four-wave mixing (FWM) causes the generation of two new frequencies (called Stokes and anti-Stokes waves, depending upon their energy content), following energy conservation rules $\left(f_{S}=2 f_{I}-f_{K}, f_{A}=2 f_{K}-f_{I}\right)$. The power of the Stokes or anti-Stokes waves oscillates along the fibre with a spatial frequency $\Delta \beta=2 \beta\left(\lambda_{K}\right)-\beta\left(\lambda_{I}\right)-\beta\left(\lambda_{S}\right.$ or $\left.\lambda_{A}\right)$, where $\beta$ is the propagation constant. If the wavelength difference $(\Delta \lambda)$ between the two pumps is small, the term $\Delta \beta$ is directly related to the local chromatic dispersion $(D)$ of the fiber by the following equation:

$$
\Delta \beta=\frac{2 \pi}{\Lambda}=\frac{\pi \cdot c}{\lambda^{2}} \cdot D \cdot \Delta \lambda^{2}
$$

where $c$ is the speed of light, $\Lambda$ is the spatial oscillation period, and $\lambda$ is the wavelength of the FWM pumps.

The basic idea of the local measurement of chromatic dispersion is to retrieve the power distribution of the Stokes or anti-Stokes waves along the fibre using Brillouin-optical-time-domain analysis (BOTDA). In comparison to the OTDRbased measurement [4], this method allows the use of low-power FWM pumps, thus avoiding interference from other nonlinear effects in the measurement [6]. 
For the BOTDA configuration, a spectrally narrow pump pulse needs to be launched into the fibre in the opposite direction, and tuned to a frequency that would cause Brillouin gain over the analyzed four-wave mixing product. When the anti-Stokes wave is used as a probe signal, the optical frequency of the Brillouin pump pulse has to be approximately $f_{A}+v_{B}, v_{B}$ being the Brillouin shift of the fibre. In this case, the signal obtained at the BOTDA fibre end (tuned at $f_{A}$ ) can be expressed as:

$$
\Delta P_{A}=\frac{g_{B}}{A_{e f f}} P_{A}(z) P_{P}(z) \Delta z
$$

where $g_{B}$ is the peak Brillouin linear gain, $A_{\text {eff }}$ the mode effective area, $P_{A}$ and $P_{P}$ the powers of the anti-Stokes and Brillouin pump, respectively, and $\Delta z$ the pump pulse width. The spatial oscillation of $P_{A}$ generates the same oscillation in the Brillouin signal, from which the local dispersion can be retrieved through a simple spectrogram analysis.

An important question is the choice of the Brillouin pump pulse width. While $\Delta \mathrm{z}$ is smaller than $\Lambda / 2$, larger pulses deliver a cleaner signal with large amplitude. However if $\Delta z$ becomes larger than $\Lambda / 2$, the contrast of the oscillations gradually vanishes, resulting in a loss of accuracy. Therefore, the optimum width of the Brillouin pump pulse is half of the oscillation period, in agreement with Nyquist's theorem.

\section{EXPERIMENT AND RESULTS}

The experimental setup and the wavelength arrangement are depicted in Figs 1-(a) and 1-(b), respectively. It is similar to the setup described in [6] except for the fact that several filtering devices have been added to make a spectrally more pure Brillouin pump. Two tunable, distributed feedback lasers were used to generate the FWM pumps. The first pump was simply continuous-wave, while the second one was modulated by an electro-optic modulator (EOM) to produce two nearly symmetric first-order sidebands separated approximately by the Brillouin shift $\left(v_{B}\right)$. The carrier wave was suppressed by controlling the DC bias voltage delivered to the EOM with a feedback circuit [7]. The FWM pumps were combined and amplified using a high power EDFA up to $30 \mathrm{~mW}$ for each spectral component. The longer-wavelength part of the two sidebands was used (together with the first FWM pump) to generate the initial seed for the Brillouin pump pulse by FWM in a 500-m highly nonlinear fibre (NLF) and a semiconductor optical amplifier (SOA). The NLF was inserted to acquire large amplitude of Brillouin pump by increasing the FWM efficiency. The other sideband was used as a pump for the FWM process to generate an oscillating probe signal in the fiber under test. Thus, the frequency separation between the Brillouin pump and the probe signal was constant regardless of random jitter in the frequency of the two DFB lasers and tunable by changing the modulation frequency. The polarizations of the FWM pumps were aligned parallel to achieve maximum FWM efficiency.
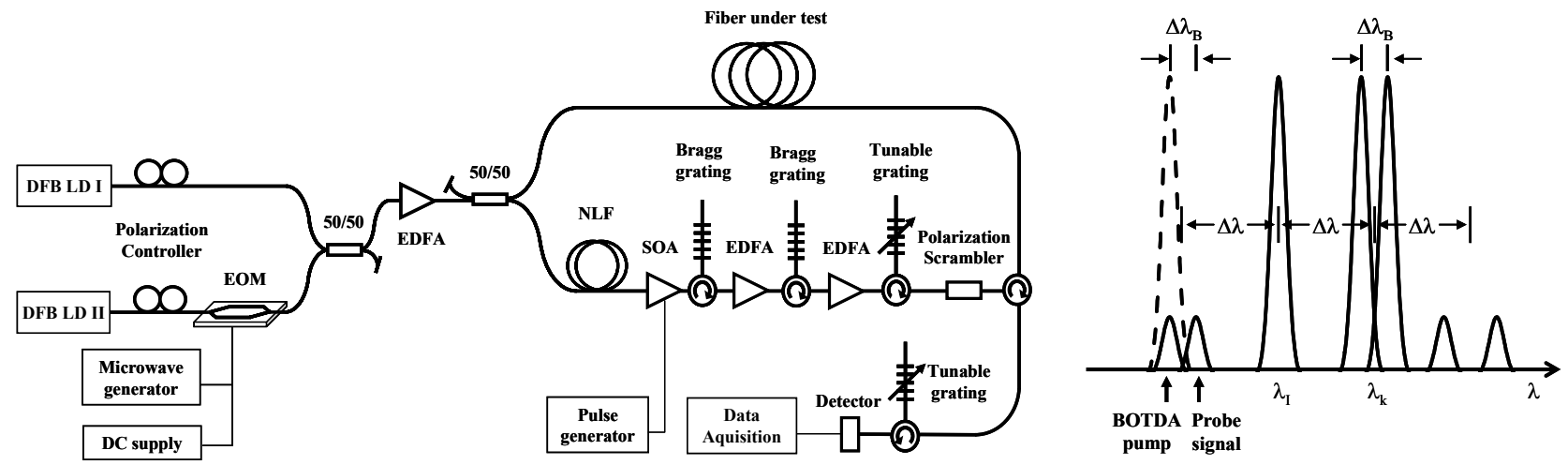

Fig. 1-(a) Experimental setup.

Fig. 1-(b) Wavelength arrangement. Pumpprobe rejection in the Brillouin pump is more than $10 \mathrm{~dB}$ better than in [6].

The Brillouin pump pulse was generated by gating the SOA using an electric pulse train, and it was subsequently filtered and amplified using Bragg gratings and EDFAs to increase its power up to a few hundred $\mathrm{mW}$. The Brillouin pump pulse was launched into the fibre in the counterpropagating direction, to produce local Brillouin gain on the probe signal along 
the fibre. This gain was measured by an optical detector (125 MHz photodiode), and a digitizing oscilloscope was used for data acquisition. The width of the pump pulse was set to half of the oscillation period of the probe signal. The polarization of the pump pulse was scrambled, and the acquired traces were averaged several thousand times to reduce the noise and ensure that no polarization-dependent gain was recorded in the trace. The frequency separation between the Brillouin pump pulse and the probe signal was swept over the region of Brillouin gain of the measured fibre and the maximum gain was recorded for each position of the fibre.

Figure 2 shows the measurement result of a conventional fibre with a chromatic dispersion of about $14 \mathrm{ps} / \mathrm{km} / \mathrm{nm}$. The length of the fibre was $1.52 \mathrm{~km}$ and the average $v_{\mathrm{B}}$ was $10.79 \mathrm{GHz}$. The wavelength of analysis $\lambda_{I}$ was $1555.2 \mathrm{~nm}$, and $\Delta \lambda$ was set to $3.17 \mathrm{~nm}$. The maximum Brillouin gain at each position is depicted as a trace in Fig 2-(a), where the inset shows the magnified view of the oscillating trace.

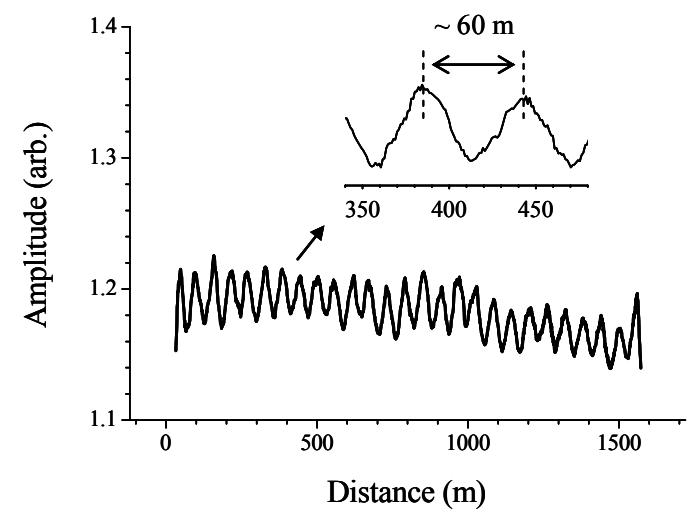

Fig. 2-(a) Trace of maximum Brillouin gain in a standard fibre, showing an oscillating period of about $60 \mathrm{~m}$.

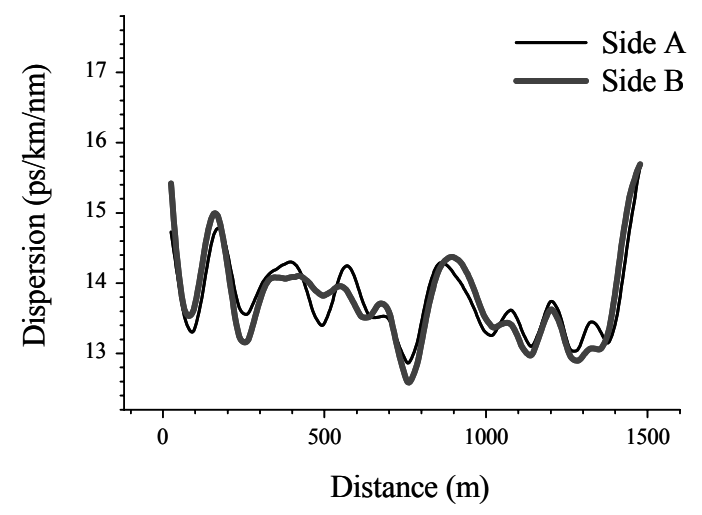

Fig. 2-(b) Local dispersion calculated from the oscillating traces in both sides. The spatial resolution (spectrogram window) is $60 \mathrm{~m}$.

We made measurements from both sides of the fibre and performed a spectrogram analysis using a window size of $60 \mathrm{~m}$. The results are depicted in Fig. 2-(b). The measurements from both sides were highly consistent, and we could observe considerable fluctuation of the local chromatic dispersion as much as \pm 1 within the length of $200 \mathrm{~m}$. Such a short period variation has never been observed in the previous lower resolution measurements, and we believe it comes from the feedback systems used in the high-speed fabrication process. The maximum difference between both sides was less than $\pm 4 \%$.

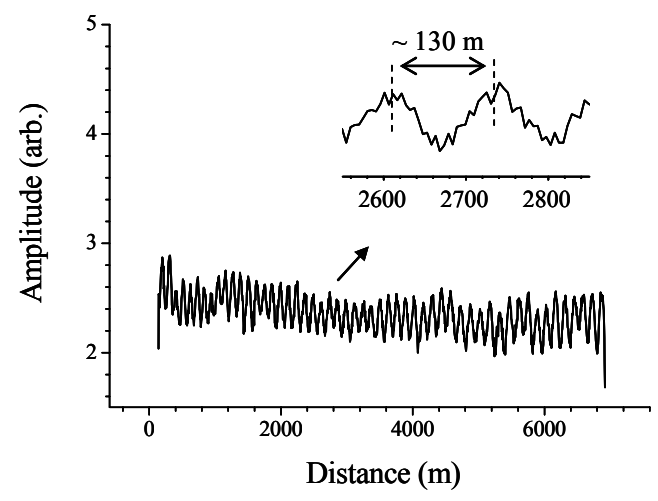

Fig. 3-(a) Trace of maximum Brillouin gain in a dispersion-shifted fibre, showing an oscillating period of about $130 \mathrm{~m}$.

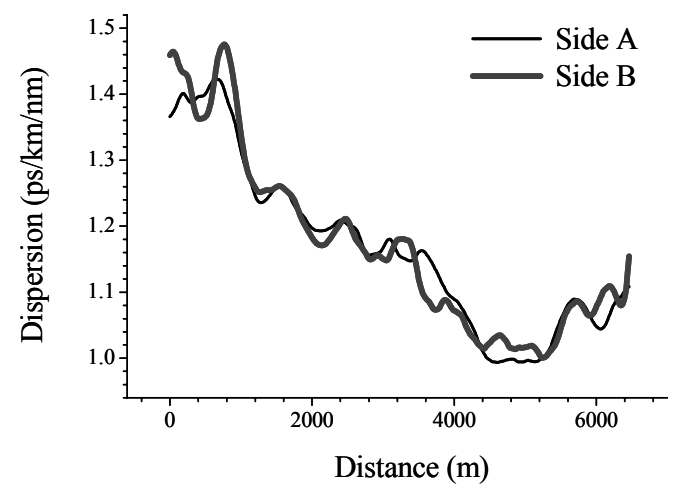

Fig. 3-(b) Local dispersion calculated from the oscillating traces in both sides. The spatial resolution (spectrogram window) is $150 \mathrm{~m}$. 
Figure 3 is the measurement result of a DSF with a chromatic dispersion of about $1.1 \mathrm{ps} / \mathrm{km} / \mathrm{nm}$. The length of the fibre was $6.7 \mathrm{~km}$ and the average $v_{\mathrm{B}}$ was $10.41 \mathrm{GHz}$. The wavelength of analysis $\lambda_{I}$ was $1559.4 \mathrm{~nm}$, and $\Delta \lambda$ was set to $7.35 \mathrm{~nm}$. The maximum Brillouin gain at each position is depicted as a trace in Fig 3-(a), where the inset shows the magnified view of the oscillating period of about $130 \mathrm{~m}$. Fig 3-(b) is the local dispersion calculated from the Fig 3-(a) with a spatial resolution of $150 \mathrm{~m}$. The maximum difference between both sides is about $\pm 6 \%$. In the case of the DSF, the spatial resolution (size of spectrogram window) was set larger than oscillating period to give more accuracy, because the signal suffered larger noise which seemed to originate from the spontaneous Brillouin scattering of FWM pumps.

Since the resolution is simply determined by the oscillating period of the trace, higher resolution is achievable if $\Delta \lambda$ is increased according to (1). However, it also needs more powerful FWM pumps or Brillouin pump to maintain the amplitude of the oscillation in the trace. In such a case, there are a few limiting factors in the resolution improvement:

1. The onset of spontaneous Brillouin scattering from the FWM pump power that results in the FWM pump depletion and a large amount of noise.

2. The gradual polarization misalignment due to the residual birefringence in the fibre for larger $\Delta \lambda$, giving rise to a decrease of the FWM efficiency.

3. The modulation instability that may deplete the Brillouin pump pulse.

These effects are more dominant in a DSF than in a standard fiber, so it seems more difficult to achieve high resolution dispersion mapping in a DSF. Although the other factors are inevitable, we believe that spontaneous Brillouin scattering of the FWM pumps may be avoidable with a proper dithering of the FWM pumps.

\section{CONCLUSION}

We demonstrated record high resolution mapping of chromatic dispersion in optical fibres based on Brillouin Optical Time Domain Analysis and four-wave mixing. The spatial resolutions of the measurements were $60 \mathrm{~m}$ and $150 \mathrm{~m}$ in a standard fibre and a dispersion shifted fibre, respectively. We expect further improvement and a resolution down to $\sim 10 \mathrm{~m}$ may be possible using this method by suppressing spontaneous Brillouin scattering of FWM pumps. This high resolution information of chromatic dispersion will be useful in several applications including parametric amplification and dispersion management in optical communication systems.

\section{REFERENCES}

1. L. F. Mollenauer, P. V. Mamyshev, J. Gripp, M. J. Neubelt, N. Mamysheva, L. Gruner-Nielsen and T. Veng "Demonstration of massive wavelength-division multiplexing over transoceanic distances by use of dispersionmanaged solitons" Opt. Lett. 25, 704-706 (2000).

2. F. Yaman, Q. Lin, S. Radic, and Govind P. Agrawal, "Impact of Dispersion Fluctuation on Dual-Pump Fiber-Optic Parametric Amplifiers,” IEEE Photon. Technol. Lett. 16, 1292-1294 (2004).

3. L. Provino, A. Mussot, E. Lantz, T. Sylvestre, and H. Maillotte, "Broadband and flat parametric amplifiers using a multi-section dispersion-tailored nonlinear fiber arrangement," J. Opt. Soc. Am. B 20, 1532-1537 (2003).

4. L. F. Mollenauer, P. V. Mamyshev and M. J. Neubelt, "Method for facile and accurate measurement of optical fiber dispersion maps" Opt. Lett. 21 1724-1726 (1996).

5. M. Eiselt, R. M. Jopson and R. H. Stolen, "Nondestructive position-resolved measurement of the zero-dispersion wavelength in an optical fiber" J. Lightwave Technol. 15 135-143 (1997).

6. M. Gonzalez-Herraez and L. Thevenaz "Simultaneous position-resolved mapping of chromatic dispersion and Brillouin shift along single-mode optical fibers" Photonics Technol. Lett. 16 1128-1130 (2004).

7. M. Nikles, L. Thévenaz and Ph. Robert "Brillouin Gain Spectrum Characterization in single-mode optical fibers" J. Lightwave Technol. 15 1842-1851 (1997). 